PROCEEDINGS OF THE

AMERICAN MATHEMATICAL SOCIETY

Volume 127, Number 6, Pages 1665-1669

S 0002-9939(99)04804-

Article electronically published on February 5, 1999

\title{
ON UPPER BOUNDS FOR HIGH ORDER NEUMANN EIGENVALUES OF CONVEX DOMAINS IN EUCLIDEAN SPACE
}

\author{
PAWEL KRÖGER
}

(Communicated by Christopher Croke)

\begin{abstract}
We derive sharp upper bounds for eigenvalues of the Laplacian under Neumann boundary conditions on convex domains with given diameter in Euclidean space. We use the Brunn-Minkowski theorem in order to reduce the problem to a question about eigenvalues of certain classes of Sturm-Liouville problems.
\end{abstract}

\section{Statement of the Results}

Let $\Omega$ be a bounded convex domain in Euclidean space $\mathbf{R}^{n}$. By considering a manifold obtained by glueing two copies of $\Omega$ together, it is possible to show that the upper bounds obtained by S.-Y. Cheng in [3] for eigenvalues of the LaplaceBeltrami operator on a Riemannian manifold yield upper bounds for the eigenvalues of the Neumann Laplacian on a bounded convex region in Euclidean space (see [5], Section 6). Our goal in this paper is to improve those bounds.

It remains an open question whether our results can be extended to Riemannian manifolds. The author was unable to replace the argument in the proof of Theorem 1 using the Brunn-Minkowski theorem by arguments related to the Bishop comparison theorem without strong additional assumptions on the cut loci of the end points of a diameter of the manifold and for other than two-dimensional manifolds (cf. section III.3 in [2] for Bishop's comparison theorem and related material).

We consider in this paper the eigenvalue problem $\triangle \phi+\mu \phi=0$ in $\Omega, \frac{\partial}{\partial n} \phi=0$ on $\partial \Omega$. The spectrum of this problem is discrete and the corresponding eigenvalues can be increasingly ordered as follows: $0=\mu_{0}(\Omega)<\mu_{1}(\Omega) \leq \mu_{2}(\Omega) \leq \ldots$. We obtain our bounds by comparison with eigenvalues of balls in Euclidean space under Dirichlet boundary conditions. It is well known that the Dirichlet eigenvalues for balls can be given explicitly in terms of zeros of Bessel functions. The positive zeros of the Bessel function $J_{\nu}$ of order $\nu$ are in increasing order $j_{\nu, 1}<j_{\nu, 2}<\ldots$ (see [1], Table 9.5 for a table of numerical values).

The following theorem gives an upper bound for $\mu_{m}(\Omega)$ in terms of the diameter of $\Omega$.

Received by the editors May 1, 1997 and, in revised form, September 2, 1997.

1991 Mathematics Subject Classification. Primary 35P15; Secondary 58G25.

(C)1999 American Mathematical Society 
Theorem 1. Suppose that $\Omega$ is a bounded convex domain in Euclidean space $\mathbf{R}^{n}$. Let $d_{\Omega}$ be the diameter of $\Omega$. Assume that $n>2$. Then,

$$
\begin{aligned}
& \mu_{m}(\Omega) d_{\Omega}^{2} \leq 4 j_{\frac{n-2}{2}, \frac{m+1}{2}}^{2} \text { if } m \text { is odd and } \\
& \mu_{m}(\Omega) d_{\Omega}^{2} \leq\left(j_{\frac{n-2}{2}, \frac{m}{2}}+j_{\frac{n-2}{2}, \frac{m+2}{2}}\right)^{2} \text { if } m \text { is even. }
\end{aligned}
$$

Now assume that $n=2$. Then,

$$
\mu_{m}(\Omega) d_{\Omega}^{2} \leq\left(2 j_{0,1}+(m-1) \pi\right)^{2} .
$$

Remarks. 1. The upper bound for $\mu_{1}(\Omega)$ given in Theorem 1 coincides with the bound obtained earlier by Cheng in [3], Theorem 2.1.

2. We have $4 j_{\nu, \frac{m+1}{2}}^{2}=\pi^{2} m^{2}+o\left(m^{2}\right)$ for $m$ large and $\nu$ fixed. Thus, it is easy to see that our bound is, at least for $m$ large, sharper than the following explicit upper bound for $\mu_{m}(M)$ obtained in [3] for manifolds with diameter $d_{M}$ and Ricci curvature bounded below by zero: $\mu_{m}(M) \leq \frac{\left(j_{\frac{n}{2}-1}\right)^{2} \times 4 m^{2}}{\left(d_{M}\right)^{2}}$ where $j_{\frac{n}{2}-1}$ is the first zero of the $\left(\frac{n}{2}-1\right)$-st Bessel function. Obviously, $j_{\frac{n}{2}-1}>\frac{\pi}{2}$ for every $n \geq 2$. Actually, an application of Sturm's comparison theorem similar to that in the proof of Proposition 2 shows that our bounds are sharper than the bounds obtained in [3] for every $m>1$. We prefer to deduce this fact from Remark 3.

3 . We can easily see from the proof of the proposition that the bounds for $\mu_{m}(\Omega)$ obtained in the above theorem are sharp for $n>2$ by considering sets obtained by glueing an appropriate pair of thin cones together. A maximizing sequence for $n=2$ and $m>1$ can be obtained by glueing a thin rectangle and two triangles together. We notice that there are many essentially different maximizing sequences for $n=3$ and $m \geq 2$ (cf. the discussion at the end of the proof of Proposition 2).

The proof of the theorem is based on the following proposition:

Proposition 2. Let $\mu_{m}(f)$ for $m=0,1,2, \ldots$ be the $m$-th Neumann eigenvalue of the following Sturm-Liouville problem on $(0,1)$ :

$$
\psi^{\prime \prime}(r)+\frac{d}{d r}(\ln f(r)) \psi^{\prime}(r)+\mu \psi(r)=0
$$

where $f^{1 /(n-1)}$ is a positive and concave function on the interval $(0,1)$. Assume that $n>2$. Then,

$$
\begin{aligned}
& \mu_{m}(f) \leq 4 j_{\frac{n-2}{2}, \frac{m+1}{2}}^{2} \text { if } m \text { is odd and } \\
& \mu_{m}(f) \leq\left(j_{\frac{n-2}{2}, \frac{m}{2}}+j_{\frac{n-2}{2}, \frac{m+2}{2}}\right)^{2} \text { if } m \text { is even. }
\end{aligned}
$$

Now assume that $n=2$. Then,

$$
\mu_{m}(f) \leq\left(2 j_{0,1}+(m-1) \pi\right)^{2} .
$$

Remark. We will make extensive use of variational characterizations of eigenvalues (cf. [4], Sect. I.4.6 and VI.1). In particular,

$$
\mu_{m}(f)=\inf _{X} \sup _{\psi \in X} \frac{\int_{0}^{1}\left|\psi^{\prime}(r)\right|^{2} f(r) d r}{\int_{0}^{1}|\psi(r)|^{2} f(r) d r}
$$

where the infimum is taken over all $(m+1)$-dimensional linear subspaces $X$ of sufficently smooth functions with compact support in $(0,1)$. We notice that we do not need an explicit boundary condition for the case of Neumann eigenvalues which is considered here (cf. [4], Sect. IV.5.1). 


\section{Proofs}

Proof of Proposition 2. Let $\psi_{m}$ be a Neumann eigenfunction for the eigenvalue $\mu_{m}(f)$ of (1). We can normalize $f$ by assuming that $\sup _{r \in(0,1)} f(r)=1$. Thus, the set of the restrictions to $[\epsilon, 1-\epsilon]$ of all $f$ satisfying the assumptions of the proposition is a compact subset of the space of all Lipshitz continuous functions on $[\epsilon, 1-\epsilon]$ with the natural norm for every positive $\epsilon<\frac{1}{2}$. Moreover, the set of eigenvalues $\mu_{m}(f)$ for all problems of the above type is uniformly bounded for every $m$ (cf. [3]). We can conclude from standard theorems on the continuous dependence of solutions of Sturm-Liouville equations on the coefficients that there is a function $f_{\max }$ satisfying the assumptions of the above theorem such that $\mu_{m}(f)$ attains its maximum value for $f=f_{\max }$. We aim to identify $f_{\max }$.

We set $g \equiv f^{1 /(n-1)}$. Since $g$ is positive and concave on $(0,1)$,

$$
g \geq \max \left\{r g^{\prime} ;(r-1) g^{\prime}\right\} \quad \text { for every } r \in(0,1) .
$$

This leads in particular to $2 g-(2 r-1) g^{\prime}>0$ for every $r \in(0,1)$.

We introduce a function $G$ on $(0,1)$ by

$$
G(r) \equiv \frac{g^{\prime}}{2 g-(2 r-1) g^{\prime}} .
$$

By $(2),-1 \leq G(r) \leq 1$ for every $r$.

It is easy to check that $G$ is constant on every interval where $g=f^{1 /(n-1)}$ is linear: $g(r)=a+b r$ on $\left(r_{1}, r_{2}\right)$ for constants $a$ and $b$ yields $G(r)=\frac{b}{2(a+b r)-(2 r-1) b}=$ $\frac{b}{2 a+b}$. A straight-forward computation shows that the assumption that $g=f^{1 /(n-1)}$ is concave is equivalent to the property that $G$ is decreasing.

We recall that $u \equiv \frac{\psi_{m}^{\prime}}{\psi_{m}}$ is a solution of the following Riccati equation:

$$
u^{\prime}+u^{2}+\frac{d}{d r}(\ln f(r)) u+\mu_{m}=0
$$

Standard comparison theorems for first order differential equations applied to this Riccati equation on an interval where $\psi \neq 0$ yield that the product $\frac{d}{d r}(\ln f(r)) \operatorname{sign}(u)$ must be as small as possible for every $r$ in that interval if $\mu_{m}$ is maximal (see [7], Theorem 3.4.1). Now we are ready to identify those $G$ which correspond to functions $f_{\max }$ which maximize $\mu_{m}$. Obviously,

$$
G(r)=\frac{1}{\frac{2(n-1)}{\frac{d}{d r}(\ln f(r))}-(2 r-1)}
$$

increases if $\frac{d}{d r}(\ln f(r))$ increases for every $r$. Hence, $G$ must be as small as possible for $u>0$ and as large as possible for $u<0$. This shows in particular that $G(r)=1$ for $0<r<r_{1}$ where $r_{1}$ denotes the smallest $r>0$ with $\psi_{m}(r)=0$. Assume now that $\psi_{m}$ is an eigenfunction corresponding to the maximal eigenvalue $\mu_{m}$ and that $\psi_{m} \neq 0$ on $\left(r_{2}, r_{3}\right)$ with $0<r_{2}<r_{3}<1$. There is an $r_{0} \in\left(r_{2}, r_{3}\right)$ such that $u=\frac{\psi_{m}^{\prime}}{\psi_{m}}$ is positive on $\left(r_{2}, r_{0}\right)$ and negative on $\left(r_{0}, r_{3}\right)$. If we take into account that every admissible function $G$ is decreasing, we can conclude that $G$ is constant on $\left(r_{2}, r_{3}\right)$. We have shown that a function $G$ corresponding to $f_{\max }$ is constant on every interval where $\psi_{m} \neq 0$. 
Assume that $G(r)=\frac{1}{\frac{2(n-1)}{d r(\ln f(r))}-(2 r-1)}=C$ for a constant $C \neq 0$ with $-1 \leq C \leq$ 1. In order to simplify the notation, we will only consider the case $C>0$. Thus,

$$
\frac{d}{d r}(\ln f(r))=\frac{n-1}{r+\frac{1}{2}\left(\frac{1}{C}-1\right)} .
$$

We set $\nu \equiv(n-2) / 2$ and

$$
z_{\nu}(r) \equiv\left(r-\frac{1}{2}\left(\frac{1}{C}-1\right)\right)^{\nu} \psi_{m}\left(\left(\mu_{m}\right)^{-1 / 2}\left(r-\frac{1}{2}\left(\frac{1}{C}-1\right)\right)\right) .
$$

By eqn. (1), $z_{\nu}$ is a solution of the Bessel equation

$$
\left(r^{1 / 2} z_{\nu}\right)^{\prime \prime}+\left(1-\left(\frac{\nu^{2}-\frac{1}{4}}{r^{2}}\right)\right) r^{1 / 2} z_{\nu}=0
$$

on every interval where $G$ is constant.

The condition that $\mu_{m}$ is maximal leads to the condition that the difference of two consecutive zeros of $z_{\nu}$ is as large as possible (take into account that $\psi_{m}$ is defined on the fixed interval $(0,1)$ and that the number of zeros of $\psi_{m}$ coincides with the order $m$ of the eigenvalue $\mu_{m}$ (see [4], Sect. VI.6)). A standard application of Sturm's comparison theorem to the Bessel equation (4) shows that the difference between two consecutive zeros of a cylindrical function $Z_{\nu}$ (recall that a cylindrical function $Z_{\nu}$ is a solution of $(4)$ on $\left.(0, \infty)\right)$ is a decreasing function of the first of the two zeros for $\nu>1 / 2$ and an increasing function of the first of the two zeros for $0 \leq \nu<1 / 2$.

First, we consider the case $\nu=(n-2) / 2>1 / 2$. We can conclude that there is an $r^{*} \in(0,1)$ with $\psi_{m}\left(r^{*}\right)=0$ such that $G(r)=1$ for $r<r^{*}$ and $G(r)=-1$ for $r>r^{*}$. Thus, $f_{\max }(r)=\left(r / r^{*}\right)^{n}$ for $r<r^{*}$ and $f_{\max }(r)=\left((1-r) /\left(1-r^{*}\right)\right)^{n}$ for $r>r^{*}$. If we take into account that the sequence $l \mapsto j_{\frac{n-2}{2}, l+1}-j_{\frac{n-2}{2}, l}$ is decreasing (this follows immediately from the above statement about the zeros of $Z_{\nu}$ ), we are led to $r^{*}=\frac{1}{2}$ if $m$ is odd and $r^{*}=\frac{j_{\frac{n-2}{2}, \frac{m}{2}}}{j_{\frac{n-2}{2}, \frac{m}{2}}+j_{\frac{n-2}{2}}, \frac{m+2}{2}}$ if $m$ is even (obviously, we could replace $r^{*}$ by $\left.1-r^{*}\right)$.

We recall now that the difference of two consecutive zeros of $Z_{\nu}$ tends to $\pi$ for every $\nu$ if the zeros tend to infinity. Assume that $n=2$ and $\nu=0$. We obtain that the difference of two consecutive zeros of $Z_{0}$ is always less than $\pi$. However, the difference of two consecutive zeros of $\psi_{m}$ is equal to $\left(\mu_{m}\right)^{-1 / 2} \pi$ if $G \equiv 0$ on the corresponding interval. Recall that we have denoted the smallest positive zero of $\psi_{m}$ by $r_{1}$ and that $G(r)=1$ for $0<r<r_{1}$. We conclude that $f_{\max }(r)=r / r_{1}$ for $r \in\left(0, r_{1}\right)$, that $f_{\max }(r)=1$ on $\left(r_{1}, 1-r_{1}\right)$, and that $f_{\max }(r)=(1-r) /\left(1-r_{1}\right)$ for $r \in\left(1-r_{1}, 1\right)$. Finally, $r_{1}=\frac{j_{0,1}}{2 j_{0,1}+(m-1) \pi}$.

The case $n=3$ plays an exceptional role since every cylindrical function has the form $Z_{3}(r)=\omega r^{-1 / 2} \sin (r+\alpha)$ for constants $\alpha$ and $\omega$. Hence, any $f_{\max }$ with $f_{\max }^{1 / 2}$ concave such that the restriction of $f_{\max }^{1 / 2}$ to $\left[\frac{k}{m+1}, \frac{k+1}{m+1}\right]$ is linear for every integer $k$ with $0 \leq k \leq m$ works.

Proof of Theorem 1. We consider a pair of points $x_{0}, x_{1}$ in $\Omega$ such that dist $\left(x_{0}, x_{1}\right)$ $=d_{\Omega}$. Obviously, $x_{0}$ and $x_{1}$ belong to $\partial \Omega$. Moreover, $\Omega$ is contained in the slab between the planes through $x_{0}$ and $x_{1}$ which are perpendicular to the line segment connecting those two points. Let $P_{r}$ for $0 \leq r \leq 1$ be the plane with dist $\left(x_{0}, P_{r}\right)=$ $r d_{\Omega}$ and dist $\left(x_{1}, P_{r}\right)=(1-r) d_{\Omega}$. Let $f(r)$ be the $(n-1)$-dimensional Lebesgue 
measure of the intersection $P_{r} \cap \Omega$. The Brunn-Minkowski theorem (see [6]) shows that $f$ satisfies the assumptions of Proposition 2.

Let $\psi_{0}, \ldots, \psi_{m}$ be eigenfunctions for the first $m+1$ Neumann eigenvalues of the Sturm-Liouville equation (1). Assume that $\psi=c_{0} \psi_{0}+\ldots+c_{m} \psi_{m}$ for real constants $c_{0}, \ldots, c_{m}$. Integration by parts yields that

$$
\int_{0}^{1} \psi^{\prime}(r)^{2} f(r) d r \leq \mu_{m}(f) \int_{0}^{1} \psi(r)^{2} f(r) d r .
$$

We set

$$
\phi(x) \equiv \psi(r) \quad \text { for } x \in P_{r} \text { and } 0<r<1 .
$$

Then,

$$
\begin{aligned}
& \int_{\Omega} \phi(x)^{2} d V(x)=d_{\Omega} \int_{0}^{1} \psi(r)^{2} f(r) d r \quad \text { and } \\
& \int_{\Omega}|\nabla \phi(x)|^{2} d V(x)=d_{\Omega}^{-1} \int_{0}^{1} \psi^{\prime}(r)^{2} f(r) d r .
\end{aligned}
$$

Hence,

$$
\int_{\Omega}|\nabla \phi(x)|^{2} d V(x) \leq d_{\Omega}^{-2} \mu_{m}(f) \int_{\Omega} \phi(x)^{2} d V(x)
$$

for arbitrary real constants $c_{0}, \ldots, c_{m}$. Using the linear independence of $\psi_{0}, \ldots, \psi_{m}$, we can conclude from the variational characterization of the eigenvalues of the Laplacian that

$$
\mu_{m}(\Omega) d_{\Omega}^{2} \leq \mu_{m}(f)
$$

The assertion of the theorem follows from Proposition 2.

\section{ACKNOWLEDGMENT}

The author is greatly indebted to Professor Rodrigo Bañuelos for many stimulating discussions and for his hospitality at Purdue University where this work was done.

The author is very grateful to the referee for his critical comments on a previous version of the paper.

\section{REFERENCES}

[1] Abramowitz, M.; Stegun, I. A., Handbook of mathematical functions. Dover, New York 1964. MR 34:8606

[2] Chavel, I., Eigenvalues in Riemannian geometry. Academic Press, Orlando 1984. MR 86g:58140

[3] Cheng, S.-Y., Eigenvalue comparison theorems and its geometric applications. Math. Z. 143, 289-297 (1975). MR 51:14170

[4] Courant, R.; Hilbert, D., Methods of mathematical physics, vol. I. Interscience, New York 1953. MR 16:426a

[5] Davies, E. B., Spectral properties of compact manifolds and changes of metric. Amer. J. Math. 112, 15-39 (1990). MR 90k:58232

[6] Federer, H., Geometric measure theory. Springer, New York 1969. MR 41:1976

[7] Hartman, P., Ordinary differential equations. John Wiley \& Sons, Baltimore 1973. MR 49:9294

Department of Mathematics, Temple University, Philadelphia, Pennsylvania 19122 Current address: Departamento de Matemática, Universidad Técnica Federico Santa María, Valparaiso, Chile

E-mail address: pkroeger@mat.utfsm.cl 